\title{
The Application of Emotional Design in the Web Interface
}

\author{
Cheng Qian ${ }^{1}$, Dehong Tang ${ }^{2}$ \\ 1,2 Hubei University of Technology, Wuhan, Hubei, China, 430068
}

\section{KEYWORDS: Emotional Design, Web Interface, Application}

\begin{abstract}
In today's rapid economic development, people's lives have been limited to the pursuit of material conditions, but the pursuit of the spirit of the pursuit of a higher level, emotional design came into being, its design has been filed in many areas. Study on interface design with high development in recent years, the Internet is more popular field of study, therefore, these two trends illustrate the emotional design study will import the web interface is needed.
\end{abstract}

\section{Introduction}

Relationship and function of the product has always been more concerned about the issue. People in the design of a product, it tends to focus most of its functions, while ignoring the feelings between the user and the product. At any time scientific and technological development, improvement of living standards, not the people to adapt to the product, but the product to adapt to people, people to pursue not only stay on the substance, but the pursuit of a higher level of spiritual and emotional experience.

When people living in the material are met, then the pursuit of emotion, fun has become the basic direction of modern living trends, pursuit of spiritual life is to enjoy the rights enjoyed by everyone. Plato said, "Human emotion is like a carriage with two horses, one on behalf of reason and another horse that is emotion. When consumers buy goods only reason foal, and emotions are high horse." Consumers in the consumer product experience will be touched by the emotional pursuit and satisfaction will bring a special role to the product design. Emotional design is conveyed to consumers to stimulate some kind of emotional information, the designer through the emotions caused by the design of certain psychological needs of consumers, through the emotional factors to control people's consumption behavior. So as a designer, you have to fully respect the consumer, to meet consumer demand, stimulate feelings of consumers, for consumers to bring more joy to meet the moving and emotional.

In the global information technology today, the network has been closely linked with our lives. Availability and ease of use has become the most basic requirements of web interface design, people began to pursue a higher level of demand for the network interface, such as emotion. Interface design advocates focus on user experience, we have a "user-centric" design, the ultimate goal of these ideas and emotional design are from the user's perspective, satisfying the customer's emotional needs, and finally allows the user to obtain psychological pleasure to meet and emotional.

\section{The Definition of Emotional Design}

Emotional design pays attention to design of humanity, in addition to meet the product functionality, but also people-centered, giving products more humane to meet the spiritual needs of the users. Designers in the design of a work time, with a certain purpose is to carry out creation, emotional 
behavior for people to have a great driving force to become an entry point for designers. If you can focus on the needs of consumers, stimulate different emotions, not only to meet consumer emotional experience you can also make the design more suitable for the purposes of need. Emotional design is the designer through the required emotion into the design works to stimulate the user's emotion to enjoy using the process to get the spirit of joy and satisfaction, and ultimately get the consumer to buy or improve efficiency purposes.

\section{Three Levels of Emotional Design}

Professor Norman, Professor Andrew and professor Witham in the study of emotions in the show: All kinds of human property by different levels of the brain caused by the automatic pre-set level, said the instinct level; section contains disposable daily behavior of brain activity, called behavior level; brain thinking part, said the level of reflection, instinctive level so quickly, it can quickly make a judgment on good or bad, safe or dangerous, appropriate signals to the muscles (motor system) issued a warning to other brain section; this is the starting point for emotional processing, as determined by biological factors may be to enhance or suppress them via a control signal: the level of behavior is where the majority of human behavior, it can reflect on the level of activity to enhance or inhibit, in turn, it also may enhance or inhibit visceral level. The highest level is a reflection of the level of reflection. It is worth noting that with sensory inputs and behavioral control no direct access, reflect and try to make the level of behavior have some bias.

Layer instinct is to bring people the most direct stimulus on the senses, like a beautiful picture. Visceral level design requires visual artists as well as industrial engineers and drafting skills, shape and form are important, physical feel and texture of the material is important, weight is also important; visceral level design say is immediate emotional impact. Must be feeling comfortable, it looks good, sexy and sensual features at work. After Apple's poor performance in the case, the introduction of color covers iMac computers, Apple iMac configuration compared to other models of the configuration is almost the same, only in appearance made some changes in the company's sales will grow rapidly.

Layer behavior is that the user must learn to master the methods and skills, and in this way to solve problems, to get a sense of accomplishment, or some other emotion in the process of solving problems. Behavior level design work is effective. In fact, the shape is not important here, the design principle is not important, important it is performance. Four excellent behavior level designs: functionality, understandability, availability and physical feeling.

Reflection layer is the highest level of consciousness, emotion and cognition, it is designed to reflect a deeper emotional user generated heart, awareness, understanding, personal experience, cultural background influence various intertwined caused. Reflection level design includes many areas and its focus is the meaning of information, culture and product or product utility. At this level, mainly due to the impact of the first two levels, and in the heart of the user generated.

\section{The Theoretical Support of Emotional Design in the Web Interface}

Professor Donald Norman wrote in emotional design: in the 1980s, when I wrote "design psychology," a book, and did not - take into account the emotional, the book focuses on the use and availability; function and form, these discussions are logical, not doping of any passion) even when it comes to design some very poorly when items, too, but now I have changed, and why in part because the brain, emotional and cognitive mutual Effects of other aspects of the progress in recent years with the development of science and technology available, scientists now realize that in 
everyday life, how important emotion, how valuable, of course, the use and availability is still very important, but there is no fun and fun, joy and excitement and even anxiety and fear, fear and anger, people's lives can not be complete. By Norman this, you can learn to meet emotional advanced form, the first to meet the emotional lower level) the availability and ease of use. In the "emotional design", Professor Norman in design behavior layer also repeatedly mentioned the importance of usability, availability and ease of use to meet the consumers are aimed at better use of the product, which shows the to design user-centered design, but too much emphasis on human-centered design is likely to cause the drawbacks, so he proposed activity-centered design. When users use the network, and will form an interactive web interface, with the development of information technology, interactive design was studied as a separate subject and it shows the importance of interaction. Regardless of the availability of usability and interactive design, these are designed to meet the needs of users, giving users a good experience of the process, in order to meet the emotional needs of the user. So the theory is equivalent to the emotional needs of the user experience emotional design theory senior level, but also the guidance of these theories to achieve a theoretical final destination

\section{The Concept of Network Interface}

Web interface is built on Internet technology based on the specific user interface, along with Internet technology matures gradually formed its unique design system. Now, the Internet has made the initial data transmission, text message-based connection of computer tools, change to be able to support multimedia, can be a huge information service system to run on multiple platforms. So, making the web interface design can not simply showed only convey information, but the user should be from the emotional point of view of the network interface design. From a psychological point of view, the interface can be divided into two levels of sensory and emotional feeling, sight, touch, hearing, etc; network interface with digital technology, multimedia technology features from the technical point of view; from the communication side, the web interface has diversified, personalized, interactive, efficient, extensive propagation characteristics.

\section{The Emotional Design in Web Interface}

Navigation is usually near the top of the page where it is easy to enter the page when the user can see at first glance navigation. Navigation is critical throughout the page elements. A specific site includes many pages, and if not, then the user will find the navigation pages. This we read ordinary paperback different, because regardless of the book there is no directory, we can turn over substantially all the pages, then find the content you want to view. But web design is different, unless there is a clear link to the page, otherwise difficult for the user to view: Perhaps in this case the only user able to view the page is to guess the URL of the page. Navigation bar, like a book catalog, each page so that users can freely browse the site, while the broad sense of the capacity of the site.

Of course, in addition to the navigation bar can also be found in other ways other page of the site; such as search bar on many sites are in use, users can find the desired information or any page through it.

Earlier we had talked about manifestations of navigation in a Web page, the following we will discuss the second key element of the page, it is the interaction site. Users have been able to browse the site and eventually find the information they need, are derived from the interactive features of the site. The fundamental purpose of the site lies in the interaction using simple language and 
interesting design concept for the user to make the most rational layout and structure of the clearest use of the guide, allowing users to get emotional experience in operational use.

The appearance of the site is the best place to arouse emotions, when we see an item, first of all imprinted in our eyes is the appearance of an object, comprising an object shape, color, texture, etc., where each a variety of factors will give users some degree of emotional experience. To design a website emotional, visual web design plays an important role. / Design and a great difference between a good design is subtle, and this delicate work lies in our subconscious layers: a good design will give the user an impression but not necessarily to understand it but a great design make users excited. They will see things feel good memories will lead to even more. Web design has a characteristic taste, short and varied to attract viewers repeatedly browsing the web is important.

In the design of the site, the color has a great influence. We can use color to create a feeling, help build brand recognition site, to establish the overall theme of the site and other related elements. As with human emotions, we liked the color of things, but not necessarily a thing has a lot of colors is beautiful. The right choice in line with the site's color attribute, you will find the site of each color will play a role.

\section{Conclusion}

The emotional design used in the web interface, web interface design is not only inevitable development needs, but also to help design the interface to better meet the "user-centric" design thinking. Only from different levels to use emotional design, in order to be truly "user-centric" will really import emotional design to network interface design and to create more and more attractive network interface to users, to meet the emotional needs of users, make a balance between high-tech and high-emotion.

\section{Reference:}

[1] Wang He: Product Design Emotional Design [JJ Industrial Design in 200903.

[2] Song Mingliang. Commodity design research under the guidance of the theory of emotional design [J]. Jiangnan University .2006

[3] Zhao Chao. From a human-computer interface design to interpersonal relations [J], decorate, 2002.

[4] Huang Haiyan. Web interface visual communication research [J], decoration, 2002.08

[5] Yao ship network interface design problems and reasons [J1, Art Observation, 2003.01

[6] On Li gift packaging design emotional factors. [J]. Business culture. 200811 